\title{
Time-Distance Helioseismology Data-Analysis Pipeline for Helioseismic and Magnetic Imager Onboard Solar Dynamics Observatory (SDO/HMI) and Its Initial Results
}

\author{
J. Zhao • S. Couvidat • R.S. Bogart $\cdot$ K.V. Parchevsky • \\ A.C. Birch • T.L. Duvall Jr. • J.G. Beck • \\ A.G. Kosovichev $\cdot$ P.H. Scherrer
}

Received: 16 September 2010 / Accepted: 23 March 2011 / Published online: 4 May 2011

(C) Springer Science+Business Media B.V. 2011

\begin{abstract}
The Helioseismic and Magnetic Imager onboard the Solar Dynamics Observatory (SDO/HMI) provides continuous full-disk observations of solar oscillations. We develop a data-analysis pipeline based on the time-distance helioseismology method to measure acoustic travel times using HMI Doppler-shift observations, and infer solar interior properties by inverting these measurements. The pipeline is used for routine production of near-real-time full-disk maps of subsurface wave-speed perturbations and horizontal flow velocities for depths ranging from 0 to $20 \mathrm{Mm}$, every eight hours. In addition, Carrington synoptic maps for the subsurface properties are made from these full-disk maps. The pipeline can also be used for selected target areas and time periods. We explain details of the pipeline organization and procedures, including processing of the HMI Doppler observations, measurements of the travel times, inversions, and constructions of the full-disk and synoptic maps. Some initial results from the pipeline, including full-disk flow maps, sunspot subsurface flow fields, and the interior rotation and meridional flow speeds, are presented.
\end{abstract}

Keywords Sun: helioseismology $\cdot$ Sun: oscillations $\cdot$ Sun: SDO

\section{Introduction}

The Helioseismic and Magnetic Imager onboard the Solar Dynamics Observatory (SDO/ HMI: Schou et al., 2011) observes the solar full-disk intensity, Doppler velocity, and vector

The Solar Dynamics Observatory

Guest Editors: W. Dean Pesnell, Phillip C. Chamberlin, and Barbara J. Thompson.

J. Zhao $(\bowtie) \cdot$ S. Couvidat · R.S. Bogart · K.V. Parchevsky · J.G. Beck · A.G. Kosovichev · P.H. Scherrer W.W. Hansen Experimental Physics Laboratory, Stanford University, Stanford, CA 94305-4085, USA e-mail: junwei@sun.stanford.edu

A.C. Birch

NorthWest Research Associates, CoRA Division, 3380 Mitchell Lane, Boulder, CO 80301, USA

T.L. Duvall Jr.

Laboratory for Astronomy and Solar Physics, NASA Goddard Space Flight Center, Greenbelt, MD 20771, USA 
magnetic field of the photosphere with high spatial resolution and high temporal cadence. Similar to the Michelson Doppler Imager (MDI: Scherrer et al., 1995), an instrument onboard the Solar and Heliospheric Observatory (SOHO), the HMI Dopplergrams are primarily used for helioseismic analysis to investigate the interior structure and dynamics of the Sun. Helioseismology data-analysis pipelines are planned for near-real-time analyses of the observations in order to provide the analysis results to the helioseismology and solar physics communities. The time-distance analysis pipeline is one of the pipelines for local helioseismology studies, and other pipelines include ring-diagram analysis and far-side active region imaging. The time-distance pipeline is designed for the routine production of nearly full-disk subsurface wave-speed perturbations and horizontal flow fields every eight hours, as well as synoptic flow maps for every Carrington rotation. It can also be used to analyze specific target areas and time periods.

Time-distance helioseismology was first introduced by Duvall et al. (1993, 1996), and it has developed rapidly since then. Different inversion techniques were introduced and tested. The LSQR algorithm, introduced by Kosovichev (1996) and used later by Zhao, Kosovichev, and Duvall (2001), solves the inversion problem in the least-squares sense in the spatial domain by an iterative approach. The Multi-Channel Deconvolution (MCD) method, introduced by Jacobsen et al. (1999) and widely used in later studies (e.g. Couvidat et al., 2004), solves the least-squares problems in the Fourier domain. Later, Couvidat et al. (2005) applied a horizontal-regularization procedure for this inversion technique. More recently, an optimally localized averaging (OLA) inversion scheme was introduced to study the solar subsurface flow fields (Jackiewicz, Gizon, and Birch, 2008).

Different types of sensitivity kernels, which describe the relationship between the travel times and interior properties, were also introduced and used in the time-distance inversion problems. Kosovichev (1996) first used ray-path approximation kernels, Jensen, Jacobsen, and Christensen-Dalsgaard (2000) introduced Fresnel-zone kernels; and Birch and Kosovichev (2000), Birch, Kosovichev, and Duvall (2004), and Birch and Gizon (2007) investigated Born-approximation kernels for both sound-speed structures and flow fields. Couvidat, Birch, and Kosovichev (2006) compared subsurface sound-speed perturbation structures inferred from these different types of kernels, and found that the inversion results obtained with the different kernels were basically consistent.

Important results on the solar interior properties have been obtained from the timedistance studies as well as from other local helioseismology techniques (e.g., Komm et al., 2004; Lindsey and Braun, 2000). The introduction that follows is limited to only time-distance results due to the scope of this paper. On global scales, poleward meridional flows were found below the photosphere (Giles et al., 1997), and solar-cycle dependent meridional flow variations were also investigated and discussed (Chou and Dai, 2001; Beck, Gizon, and Duvall, 2002; Zhao and Kosovichev, 2004). On local scales, subsurface sound-speed perturbations and flow fields were derived for supergranulation (Kosovichev and Duvall, 1997; Duvall et al., 1997; Duvall and Gizon, 2000; Sekii et al., 2007; Jackiewicz, Gizon, and Birch, 2008) and for sunspots (Kosovichev, Duvall, and Scherrer, 2000; Gizon, Duvall, and Larsen, 2000; Zhao, Kosovichev, and Duvall, 2001; Couvidat, Birch, and Kosovichev, 2006; Zhao, Kosovichev, and Sekii, 2010). Additionally, time-distance helioseismology was used to detect the emergence of active regions before their appearances in the photosphere (Kosovichev, Duvall, and Scherrer, 2000; Jensen et al., 2001; Zharkov and Thompson, 2008), to image large active regions on the far side of the Sun (Zhao, 2007; Ilonidis, Zhao, and Hartlep, 2009), and to measure sound-speed perturbations in the tachocline (Zhao et al., 2009). These results are important for space-weather forecasting and understanding the mechanisms for the generation of solar magnetism. The 
time-distance helioseismology pipeline analyses, based on the high spatial-resolution and high temporal-cadence observations from HMI, will greatly advance our knowledge of the interior processes and their connections with solar activity above the photosphere.

However, one should keep in mind that the physics of solar oscillations in the turbulent magnetized plasma is very complicated, and that the helioseismic techniques are still in the process of being developed. Because of limited knowledge of the wave physics and complexity of the MHD turbulence, there may be systematic uncertainties in the local helioseismology inferences, particularly in strong magnetic-field regions of sunspots. For example, Lindsey and Braun (2005a, 2005b) argued that the outgoing and ingoing travel-time asymmetries observed in sunspot areas might be caused by a "shower-glass effect". Schunker et al. (2005) found that the inclined magnetic field in sunspot penumbrae might cause variations of measured acoustic travel times. Zhao and Kosovichev (2006) found that this inclined magnetic-field dependent effect does not exist in the measurements obtained from the MDI intensity observations. Later, Rajaguru et al. (2006) found that the non-uniform acoustic power distribution in the photosphere also contributed to measured travel-time shifts in active regions if a phase-speed filtering procedure was applied. This effect was then studied by Parchevsky, Zhao, and Kosovichev (2008) and Hanasoge et al. (2008) numerically, and also by Nigam and Kosovichev (2010) analytically. More recently, Gizon et al. (2009) derived a sunspot's subsurface flow fields after applying ridge filtering, and their inferred results did not agree with the previously inverted results with the use of phase-speed filtering. Using high spatial-resolution observations from Hinode, Zhao, Kosovichev, and Sekii (2010) showed that the principal results on sunspot structure did not depend on the use of phasespeed filtering. However, significant systematic uncertainties in sunspot seismology remain and need to be understood, and these are being actively studied by the use of numerical simulations. For a recent review of the sunspot seismology and uncertainties see Kosovichev (2010).

Despite the ongoing discussions of accuracy of time-distance measurements and interpretation of inversion results, it is useful to provide the measured travel times and the inversion results for investigations of structures and flows below the visible surface of the Sun. As the flow chart of the pipeline in Figure 1 shows, we apply phase-speed filtering to the HMI data, compute cross-covariances of the oscillations, and use two different travel-time fitting procedures to derive the acoustic travel times. We then perform inversions using two different sets of travel-time sensitivity kernels, based on the ray-path and Born approximations, to infer subsurface wave-speed perturbations and flow fields, using the MCD inversion method. We provide online access to the measured travel times, full-disk subsurface wave-speed perturbations and flow maps calculated every eight hours. In addition, we provide synoptic flow maps for each Carrington rotation. In this article, we describe details of the acoustic travel-time measurement procedure in Section 2, and the inversion procedure in Section 3. We describe the pipeline data products and present initial HMI results in Section 4, and summarize our work in Section 5.

\section{Acoustic Travel-Time Measurement}

\subsection{Tracking and Remapping}

SDO/HMI continuously observes the full-disk Sun, providing Doppler velocity, continuum intensity, line-depth, line-width, and magnetic-field maps with a 45 -second cadence, and also vector magnetic-field measurements with a cadence of 12 minutes. Each full-disk image 


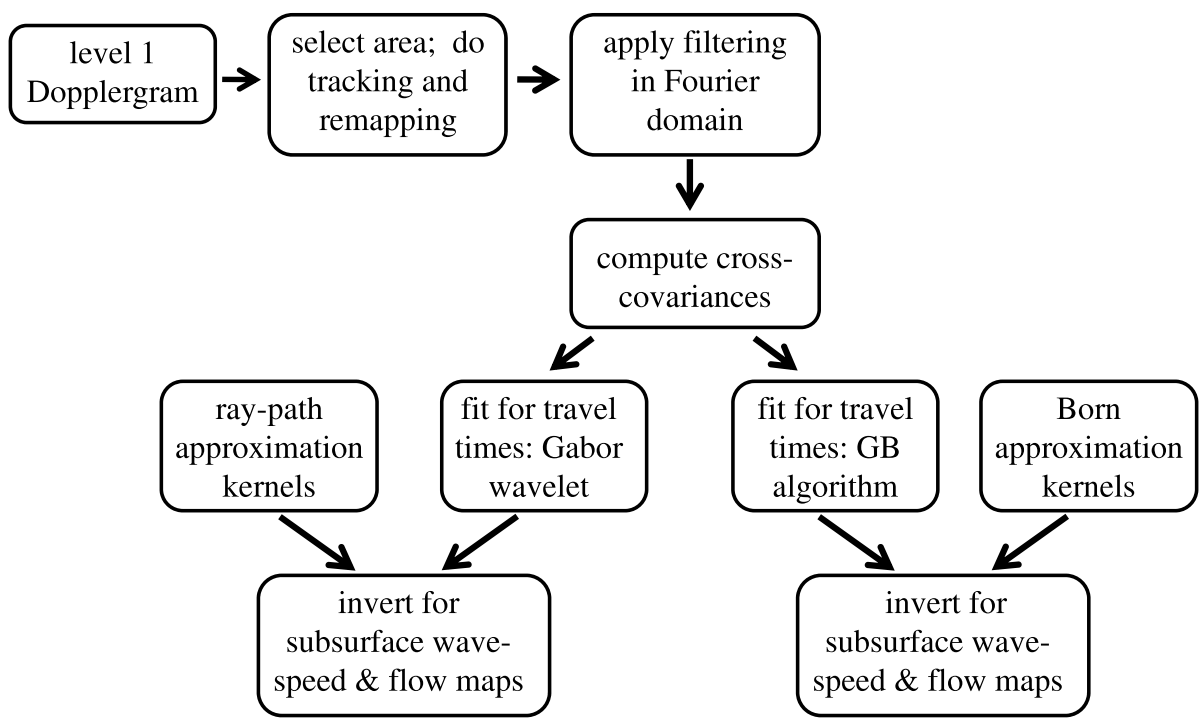

Figure 1 Flow chart for the HMI time-distance helioseismology data-analysis pipeline.

has $4096 \times 4096$ pixels with a spatial resolution of $0.504 \operatorname{arcsec}$ pixel ${ }^{-1}$ (i.e., approximately, 0.03 heliographic degree pixel $^{-1}$ at the solar disk center). The Doppler observations are primarily used for helioseismic studies. The observing sequences, algorithms for deriving Doppler velocity and magnetic field, and other instrument calibration issues, are discussed by Schou et al. (2011).

As illustrated in Figure 1, the primary input for the pipeline is Dopplergrams, although in principle, the HMI intensitygrams and line-depth data can also be analyzed in the same manner. Users of the pipeline can select specific areas for analysis, preferably within $60^{\circ}$ from the solar disk center. In practice, the users provide the Carrington longitude and latitude of the center of the selected area, and the mid-time of the selected time period, then the pipeline code selects an area of roughly $30^{\circ} \times 30^{\circ}$ centered at the given coordinate, and for a time interval of eight hours with the given time as the middle point. The data for this selected area and time period are then tracked to remove solar rotation, and remapped into the heliographic coordinates using Postel's projection (also known as azimuthal equidistant projection) relative to the given area center. Normally, the tracked area consists of $512 \times 512$ pixels with a spatial sampling of $0.06^{\circ}$ pixel $^{-1}$; and the temporal sampling is the same as the observational cadence. Cubic interpolation is used for the pixels not located on the observational grid.

Figure 2 shows typical HMI $k-\omega$ and time-distance diagrams obtained from one tracked and remapped area. The selected area covers $30^{\circ}$ in latitude and has an apparent differential rotation over this span. However, for fast computations, only one uniform tracking rate corresponding to the Snodgrass rotation rate at the center of this area is used. The Snodgrass rotation rate is $2.851-0.343 \sin ^{2} \phi-0.474 \sin ^{4} \phi \mu \mathrm{rad} \mathrm{s}^{-1}$, where $\phi$ is latitude (Snodgrass and Ulrich, 1990). The uniform tracking rate of the selected area results in a differential rotation velocity in the inverted horizontal velocity fields. This differential rotation velocity is removed from the full-disk flow maps after averaging over the whole Carrington rotation, and only the residual flow fields are given as the final results (see Section 4.1). But for the user-selected areas, the differential rotation is kept in the inversion results. 
Figure 2 Typical power spectrum $(k-\omega)$ diagram (upper) and time-distance (cross-covariance) diagram (lower) made from eight hours of HMI Doppler observations.
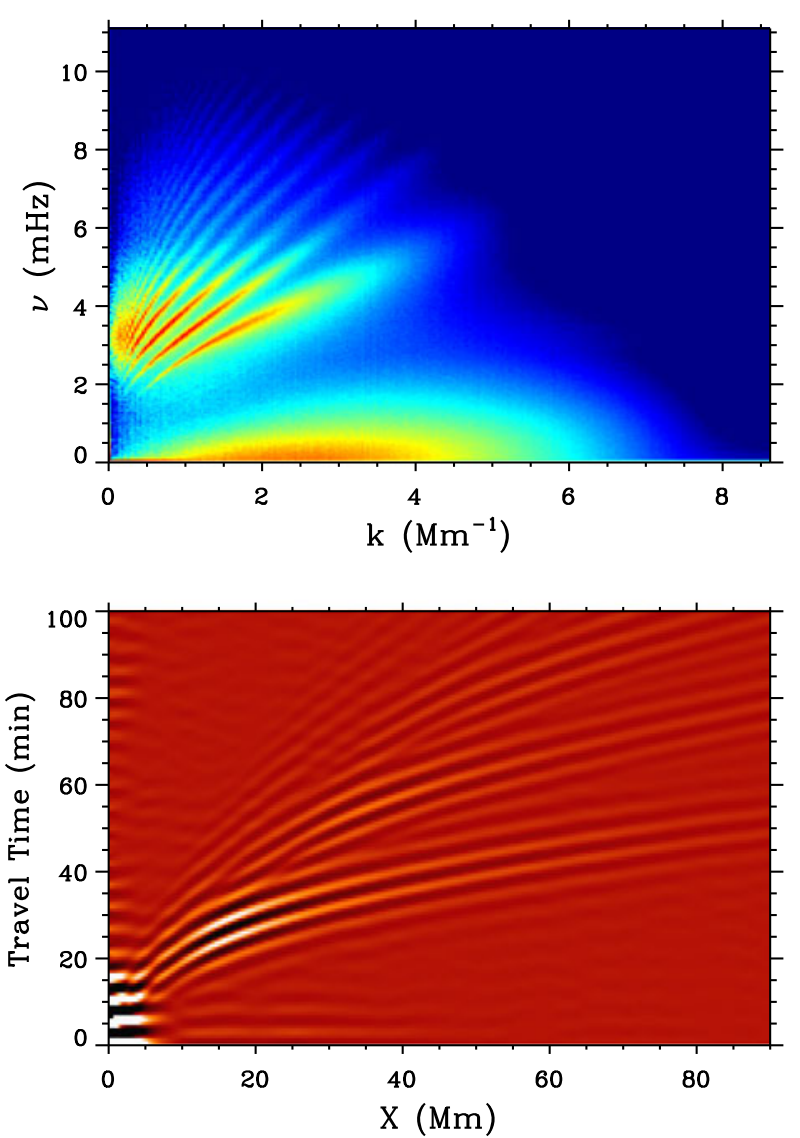

\subsection{Computing Cross-Correlations and Fitting for Travel Times}

Each tracked and remapped Dopplergram datacube is filtered in the 3D Fourier domain. Solar convection and $f$-mode oscillation signals are removed first, and then phase-speed filtering is applied following the procedures prescribed by Couvidat et al. (2005). For the travel-time measurements, for each central point we select 11 annuli with various radii and widths chosen from our past experience with MDI analyses. All of the phase-speed filtering parameters, including the central phase speed, filter width, and the corresponding inner and outer annulus radii are presented in Table 1. The phase-speed filter is a Gaussian function of the wave's horizontal phase speed. It selects wave packets traveling between the central points and the annuli for the selected distances. This filter helps improve the signalto-noise ratio, and also it removes leakage from low-degree oscillations. After the filtering, the data are transformed back to the space-time domain for cross-covariance computations. Two different fitting methods are used to derive the acoustic travel times from the crosscovariances: a Gabor-wavelet fitting (Kosovichev and Duvall, 1997), and a cross-correlation method based on seismology algorithms (Zhao and Jordan, 1998) adopted by Gizon and Birch (2002; GB algorithm hereafter). A detailed description of the filtering procedure, the cross-covariance computations, the two types of travel-time fittings, comparison of the travel times derived from the two fitting methods, and the measurement error estimates is given 
Table 1 Phase-speed filtering parameters used for the selected travel distances (annulus ranges).

\begin{tabular}{clll}
\hline Annulus No. & $\begin{array}{l}\text { Annulus range } \\
\text { (heliographic degree) }\end{array}$ & $\begin{array}{l}\text { Phase speed } \\
(\mu \mathrm{Hz} / \ell)\end{array}$ & $\begin{array}{l}\text { FWHM } \\
(\mu \mathrm{Hz} / \ell)\end{array}$ \\
\hline 1 & $0.54-0.78$ & 3.40 & 1.0 \\
2 & $0.78-1.02$ & 4.00 & 1.0 \\
3 & $1.08-1.32$ & 4.90 & 1.25 \\
4 & $1.44-1.80$ & 6.592 & 2.149 \\
5 & $1.92-2.40$ & 8.342 & 1.351 \\
6 & $2.40-2.88$ & 9.288 & 1.183 \\
7 & $3.12-3.84$ & 10.822 & 1.895 \\
8 & $4.08-4.80$ & 12.792 & 2.046 \\
9 & $5.04-6.00$ & 14.852 & 2.075 \\
10 & $6.24-7.68$ & 17.002 & 2.223 \\
11 & $7.68-9.12$ & 19.133 & 2.039 \\
\hline
\end{tabular}

by Couvidat et al. (2010). In particular, it has been shown there that the two definitions of the acoustic travel times and the two fitting methods give generally consistent results in the quiet-Sun regions, but that they may give different results in active regions. The differences are currently not well understood, but in the pipeline we implement both travel-time definitions.

In the Postel-projected maps, the exact distance between two arbitrary points cannot be calculated using the Cartesian coordinates. Thus, when an annulus is selected around a given location, some additional computations are needed to determine the exact great-circle distance between the points. The formula to determine the great-circle distance is

$$
\Delta=\arccos \left(\sin \theta_{1} \sin \theta_{2}+\cos \theta_{1} \cos \theta_{2} \cos \left(\phi_{1}-\phi_{2}\right)\right),
$$

where $\left(\theta_{1}, \phi_{1}\right)$ and $\left(\theta_{2}, \phi_{2}\right)$ are the heliospheric longitude and latitude coordinates for the two separate locations.

To facilitate the inversions for subsurface flow fields, each annulus is divided into four quadrants representing the North, South, East, and West directions (Kosovichev and Duvall, 1997). So, for each annulus and each travel-time fitting method, we obtain six measurements of acoustic travel times, corresponding to the outgoing and ingoing, East-, West-, South-, and North-going directions. We then combine these travel times to obtain one map for the mean travel time and three maps for the travel-time differences. These travel times are: $\tau_{\text {mean }}$ (average of outgoing and ingoing travel times), $\tau_{\mathrm{oi}}$ (difference of outgoing and ingoing travel times), $\tau_{\mathrm{we}}$ (difference of West- and East-going travel times), and $\tau_{\mathrm{ns}}$ (difference of Northand South-going travel times). These four travel-time maps for each annulus are archived and available through the HMI Data Record Management System (DRMS).

\section{Subsurface Wave-Speed Perturbation and Flow-Field Inversions}

The acoustic travel times are derived by two different fitting methods: the Gabor-wavelet function and the GB algorithm. Then, as illustrated in Figure 1, to infer the subsurface wavespeed perturbations and flow velocities, the Gabor-wavelet travel times are inverted using the ray-path approximation sensitivity kernels, and the GB travel times are inverted using the Born-approximation sensitivity kernels. The Born-approximation kernels are calculated based with the filter and window parameters of the GB fits. 


\subsection{Inversions}

Both the ray-path and Born-approximation kernels have been used in previous time-distance studies (see, e.g., Zhao, Kosovichev, and Duvall, 2001; Couvidat, Birch, and Kosovichev, 2006). Details of the kernel calculations and their comparisons will be given in a separate article.

We employ the MCD inversion method (Jacobsen et al., 1999) with a horizontal regularization (Couvidat et al., 2005). For the wave-speed perturbation inversions, the linearized equation relating the measured mean travel times and the subsurface wave-speed perturbations are

$$
\delta \tau_{\text {mean }}^{\lambda \mu v}=\sum_{i j k} A_{i j k}^{\lambda \mu v} \delta s_{i j k}
$$

where $\delta s_{i j k}$ is the relative wave-speed perturbation $\delta c_{i j k} / c_{i j k}$ approximated by piece-wise constant functions on the inversion grid, and $A_{i j k}^{\lambda \mu v}$ is a matrix of the discretized sensitivity kernel. Here, $\lambda$ and $\mu$ label the coordinates of the central points of the annuli in the observed areas, $v$ labels the different annuli, and $i, j$, and $k$ are the indices for the discretized threedimensional space for inversions. Usually, the horizontal coordinates of the inversion grid ( $i$ and $j$ indices) are selected at the same locations as the central travel-time measurement points ( $\lambda$ and $\mu$ indices). In the first-order approximation, the sensitivity kernels are calculated for a spherically symmetric solar model and do not depend on the position on the solar surface. Therefore, in this case Equation (2) is actually equivalent to a convolution in the horizontal domain, which can be simplified as a direct multiplication in the Fourier domain:

$$
\delta \tilde{\tau}^{v}\left(\kappa_{\lambda}, \kappa_{\mu}\right)=\sum_{k} \tilde{A}_{k}^{v}\left(\kappa_{\lambda}, \kappa_{\mu}\right) \delta \tilde{s}_{k}\left(\kappa_{\lambda}, \kappa_{\mu}\right),
$$

where $\delta \tilde{\tau}, \tilde{A}$, and $\delta \tilde{s}$ are the 2D Fourier transforms of $\delta \tau, A$, and $\delta s$, respectively; $k$ is the same as in Equation (2); $\kappa_{\lambda}$ and $\kappa_{\mu}$ are the wavenumbers in the Fourier domain corresponding to $\lambda$ and $\mu$ of the spatial domain. For each $\left(\kappa_{\lambda}, \kappa_{\mu}\right)$, the equation in the Fourier domain is a matrix multiplication:

$$
d=G m,
$$

where

$$
d=\left\{\delta \tilde{\tau}^{v}\left(\kappa_{\lambda}, \kappa_{\mu}\right)\right\}, \quad G=\left\{\tilde{A}_{k}^{v}\left(\kappa_{\lambda}, \kappa_{\mu}\right)\right\}, \quad m=\left\{\delta \tilde{s}_{k}\left(\kappa_{\lambda}, \kappa_{\mu}\right)\right\} .
$$

Thus, we have a large number of linear equations describing the depth dependence of the Fourier components, and each linear equation can be solved in the least-squares sense. After all these equations are solved, and $m$ is obtained for each $\left(\kappa_{\lambda}, \kappa_{\mu}\right)$, the values of $\delta s_{i j k}$ are calculated by the inverse 2D Fourier transform.

Equation (4) is ill-posed, and regularization is required to obtain a smooth solution. The regularized least-squares algorithm is formulated as

$$
\min \left\{\|(d-G m)\|_{2}^{2}+\lambda^{2}(\kappa)\|L m\|_{2}^{2}\right\}
$$

where $\|\ldots\|_{2}$ denotes the L2-norm, $L$ is a regularization operator, and $\lambda(\kappa)$ is a regularization parameter. We choose $L$ to be a diagonal matrix whose elements are the inverse of the square root of the spatial sampling $\Delta z$ at each depth. Such weighting is necessary because the grid in the vertical direction is chosen to be approximately uniform in the acoustic depth, 
meaning that the spatial sampling of deep layers is larger than the sampling of shallower layers. The regularization parameter is taken in the form of $\lambda^{2}(\kappa)=\lambda_{v}^{2}+\lambda_{h}^{2}(\kappa)$, where $\lambda_{v}$ and $\lambda_{h}$ are vertical and horizontal regularization parameters. The purpose of introducing $\lambda_{h}$ is to damp the high-wavenumber components that may lead to noise amplification. Following the discussion of Couvidat et al. (2005), we choose $\lambda_{h}=\lambda_{2} \kappa^{2}$ with $\lambda_{2}$ as a constant.

Because the regularization is applied in the Fourier domain, it is quite difficult to use different regularization parameters for different horizontal locations of the same region. Sometimes different regularization parameters are needed, because in active regions the noise level may be different from the quiet-Sun regions, as we discuss in Section 3.3. Thus, it is necessary to implement into the analysis pipeline another inversion technique, the LSQR algorithm, which solves Equation (2) in the space domain by an iterative approach. This implementation is currently under development.

\subsection{Inversion Depth and Validation of Inversions}

For both the wave-speed and flow-field inversions, and for both the ray-path and Bornapproximation inversions, we select a total of 11 inversion depths as follows: $0-1,1-3,3-$ $5,5-7,7-10,10-13,13-17,17-21,21-26,26-30$, and $30-35 \mathrm{Mm}$. There are a total of 11 depth intervals. The inversion results provide the wave-speed perturbations and flow velocities averaged in these layers. Due to the lack of acoustic-wave coverage in the deep interior, the reliability of inversion results decreases with the depth. Thus, only inversion results for the depths shallower than $20 \mathrm{Mm}$ are included in the pipeline output. This may change in the future when more confidence is gained in the deeper interior inversion results.

In recent years, several studies have been carried out to validate the time-distance measurements and inversions. To validate the derived subsurface flow fields, Georgobiani et al. (2007) and Zhao et al. (2007) have analyzed realistic solar-convection simulations and found satisfactory inversion results for the shallow layers covered by the simulations. Validations of the wave-speed perturbation inversions based on numerical simulations with preset structures have also been performed. Meanwhile, numerical simulations for magnetic structures with flows are also under development (Rempel, Schüssler, and Knölker, 2009; Stein et al., 2011; Kitiashvili et al., 2011). Validations of the time-distance helioseismology techniques will be carried out as well using these simulations.

Cross-comparisons between different helioseismology techniques, e.g. comparing the mean rotation speed from our pipeline analysis with global helioseismology results, and comparing the subsurface flow fields with results from ring-diagram analyses, will also be important for the validation.

\subsection{Error Estimate}

There are two types of errors in the pipeline results: systematic errors due to our limited knowledge of the wave physics in the magnetized turbulent medium and simplified mathematical formulations, and statistical errors, which are mostly due to the stochastic nature of the solar oscillations ("realization noise"). Here, we only discuss the statistical errors.

To estimate the errors in the inversion results, we need first to estimate the uncertainties in the measured acoustic travel times. Here, we estimate the measurement uncertainties following the prescriptions of Jensen, Duvall, and Jacobsen (2003) and Couvidat, Birch, and Kosovichev (2006). We select 25 quiet-Sun regions, and use the rms variation of mean travel times for different measurement distances as an error estimate for the travel times. The estimated uncertainties for the Gabor-wavelet fitting are given in Table 2, and the uncertainties obtained for the GB algorithm are similar, but slightly larger for short distances and 
Table 2 Measured mean travel times and uncertainties for both quiet-Sun regions and active regions.

\begin{tabular}{llll}
\hline Annulus No. & $\begin{array}{l}\text { Mean travel time } \\
(\min )\end{array}$ & $\begin{array}{l}\text { Uncertainty for quiet regions } \\
(\min )\end{array}$ & $\begin{array}{l}\text { Uncertainty for active regions } \\
(\mathrm{min})\end{array}$ \\
\hline 1 & 11.87 & 0.062 & 0.17 \\
2 & 18.82 & 0.061 & 0.25 \\
3 & 21.76 & 0.11 & 0.26 \\
4 & 25.85 & 0.11 & 0.19 \\
5 & 28.69 & 0.14 & 0.15 \\
6 & 31.18 & 0.14 & 0.14 \\
7 & 35.07 & 0.10 & 0.10 \\
8 & 38.86 & 0.12 & 0.11 \\
9 & 42.46 & 0.11 & 0.093 \\
10 & 46.63 & 0.14 & 0.11 \\
11 & 50.26 & 0.15 & 0.11 \\
\hline
\end{tabular}

slightly smaller for long distances. Active regions have different measurement uncertainties. To estimate these, we selected a relatively stable sunspot, NOAA AR 11092, from 2 through 5 August 2010, and we assumed that the sunspot did not change during this period. Then we use the rms of the travel times measured inside the sunspot as an error estimate. Due to the evolution of the sunspot, this approach overestimates the measurement uncertainties, but can still give us an approximate estimate of measurement errors. These error estimates are presented in Table 2 as well.

Inversions are then performed for the same quiet-Sun regions and the active region to estimate the statistical errors in inversion results. Following the same approach, the rms of inverted wave-speed perturbations is assumed as the statistical error. The error estimates for both the quiet-Sun and active regions are shown in Table 3. Because supergranular flows are dominant in the flow fields, it is difficult to estimate errors of the inverted velocity for the quiet Sun by this approach. Instead, we estimate the velocity errors based on the rotational velocity profile, which has little change in a time scale of a few days. While the errors for the wave-speed perturbation inside active regions are roughly twice those for the quiet-Sun regions, the velocity errors inside active regions sometimes are seven times larger than the errors in the quiet Sun.

\section{Data Products and Initial Results from HMI}

The time-distance data-analysis pipeline is used for the routine production of nearly realtime full-disk (actually, nearly full-disk covering a $120^{\circ} \times 120^{\circ}$ area on the solar disk) wavespeed perturbation and flow field maps every eight hours. These maps are then used to construct the corresponding synoptic maps for each Carrington rotation. The pipeline can also be used for specific target areas, such as active regions. In this section, we introduce the data products from this pipeline and some initial results from it.

\subsection{Routine Production: Full-Disk and Synoptic Maps}

For each day of HMI observations, we select three eight-hour periods: 00:00-07:59 UT, 08:00 - 15:59 UT, and 16:00-23:59 UT. For each analysis period, we select 25 regions, 
Table 3 Error estimates for the relative wave-speed perturbation and horizontal-velocity inferences in the quiet-Sun (QS) and active regions (AR).

\begin{tabular}{ccccc}
\hline $\begin{array}{l}\text { Depth } \\
(\mathrm{Mm})\end{array}$ & Wave speed for QS & $\begin{array}{l}\text { Velocity for QS } \\
\left(\mathrm{m} \mathrm{s}^{-1}\right)\end{array}$ & Wave speed for AR & $\begin{array}{l}\text { Velocity for AR } \\
\left(\mathrm{m} \mathrm{s}^{-1}\right)\end{array}$ \\
\hline $0-1$ & $2.8 \times 10^{-3}$ & 7.8 & $6.3 \times 10^{-3}$ & 58.3 \\
$1-3$ & $4.1 \times 10^{-3}$ & 7.5 & $10.9 \times 10^{-3}$ & 56.4 \\
$3-5$ & $6.4 \times 10^{-3}$ & 8.9 & $8.7 \times 10^{-3}$ & 51.1 \\
$5-7$ & $4.6 \times 10^{-3}$ & 9.4 & $9.7 \times 10^{-3}$ & 45.1 \\
$7-10$ & $4.7 \times 10^{-3}$ & 13.1 & $6.7 \times 10^{-3}$ & 34.5 \\
$10-13$ & $3.7 \times 10^{-3}$ & 12.9 & $3.1 \times 10^{-3}$ & 28.1 \\
\hline
\end{tabular}

Figure 3 Schematic plot showing how areas are selected for a routine calculations of the full-disk wave-speed and flow maps. Not all of the 25 selected areas are shown.

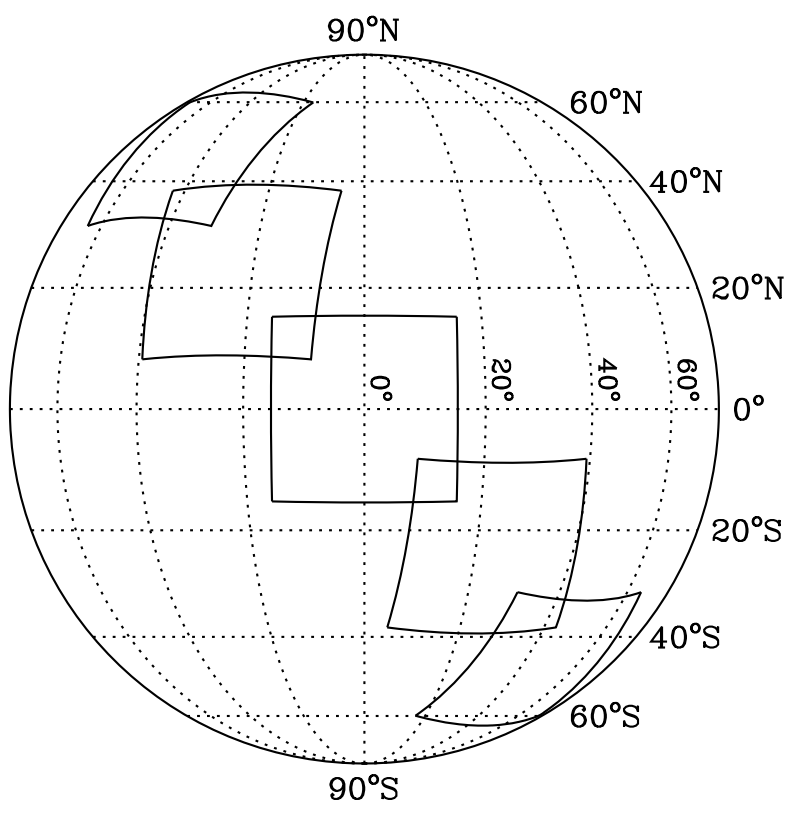

with the central locations at $0^{\circ}, \pm 24^{\circ}$, and $\pm 48^{\circ}$ in both longitude and latitude, where the longitude is relative to the central meridian at the mid-time of the selected period. Figure 3 shows locations of these areas on the solar disk. The total number of areas is 25 : five rows and five columns. Due to the Postel's projection, the boundaries of these areas are often not parallel to the latitude or longitude lines. It is also evident that many areas overlap, some areas overlap twice, and some overlap four times. The travel times and inversion results are averaged in these overlapped areas. However, in the areas close to the solar limb, the foreshortening effect may become non-negligible, but the role of this is not yet systematically studied. Users of these maps are urged to be cautious when using the pipeline results in the areas close to the limb.

For each full-disk map and each synoptic map, the East-West velocity [ $\left.v_{x}\right]$, the NorthSouth velocity $\left[v_{y}\right]$, and wave-speed perturbation $[\delta c / c]$ in each depth layer are derived with a horizontal spatial sampling of $0.12^{\circ} \operatorname{pixel}^{-1}$. For each of the 25 areas, the inversion results are first obtained in the Postel-projection coordinates, and then converted into the 


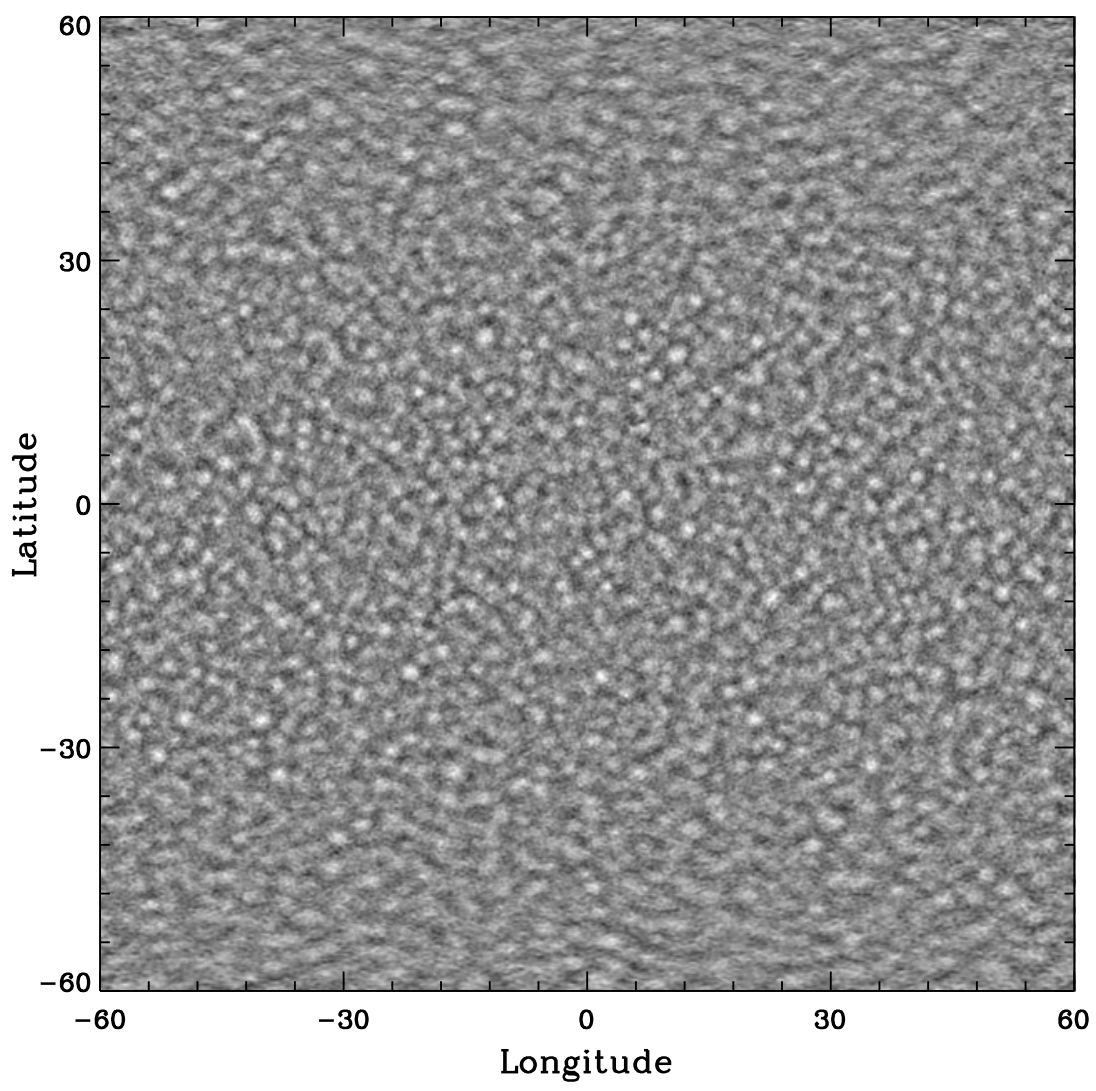

Figure 4 A map of horizontal flow divergence for a depth range of 1-3 Mm and a time period of 00:00-07:59 UT 19 May 2010. The display scale is from $-6.2 \times 10^{-4}$ to $6.2 \times 10^{-4} \mathrm{~s}^{-1}$. White areas with positive divergence correspond to supergranulation. Note that supergranules appear larger at high latitudes because of the rectangular longitude-latitude map projection.

longitude-latitude coordinates. This coordinate conversion is basically the inverse procedure of transforming the observed data into the Postel-projection coordinates for the travel-time measurements. Cubic spline interpolation is employed. The results in high-latitude regions are oversampled. After the coordinate transformation, the overlapped areas are averaged, and the final statistical errors are estimated for the whole procedure starting from the traveltime measurements. This includes all potential errors from the interpolation and remapping. The final full-disk results are saved on a uniform longitude-latitude coordinate grid, so each horizontal image of the subsurface layers has a total of $1000 \times 1000$ pixels covering $120^{\circ}$ in both longitudinal and latitudinal directions. This coordinate choice is convenient for merging and analyzing results, but unavoidably distorts maps in high latitude areas.

Figure 4 shows an example of a full-disk map of the subsurface horizontal flow divergence calculated from $v_{x}$ and $v_{y}$ at the depth range of 1-3 Mm. The positive-divergence areas correspond to supergranulation. Such maps at various depths with continuous temporal coverage can be valuable for studying the structure and evolution of the supergranulation. Figure 5 displays the subsurface horizontal flow fields with full spatial resolution for a region located at the center of the map in Figure 4. Supergranular flows can be easily identified. 


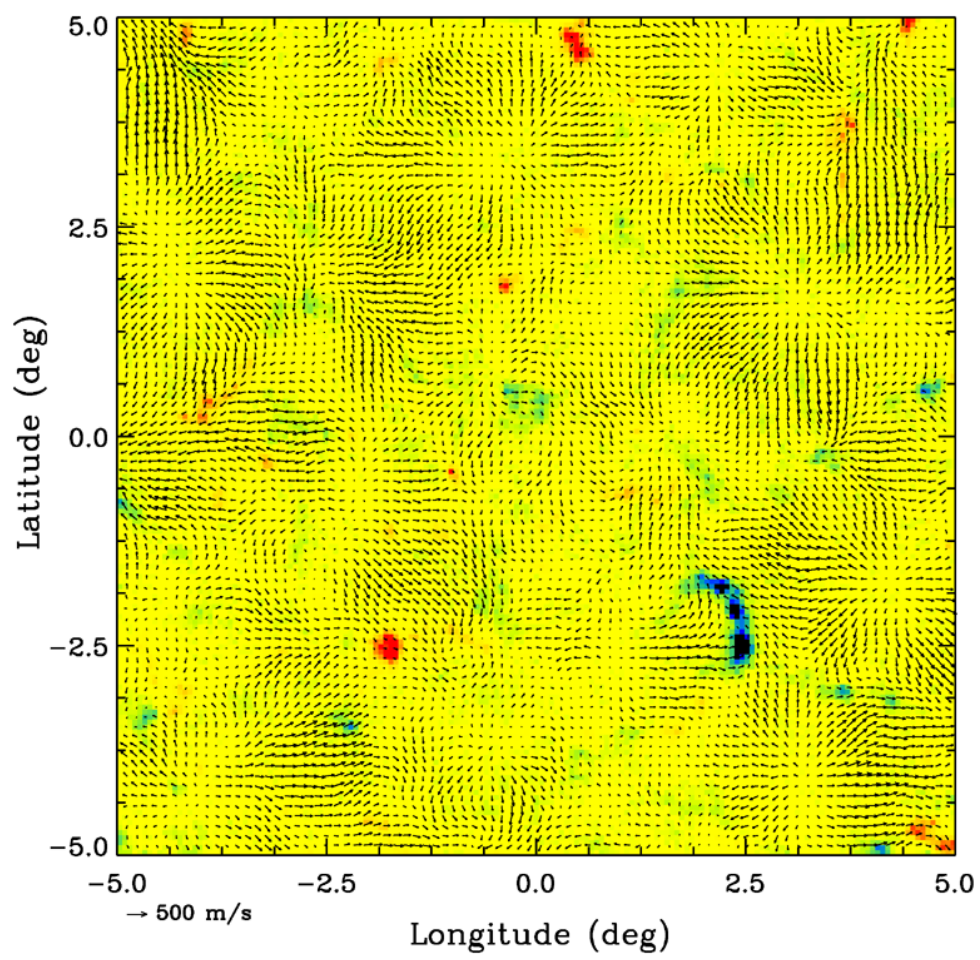

Figure 5 A sample of subsurface horizontal flow fields with full spatial resolution at the depth of $0-1 \mathrm{Mm}$. This area is sampled at the center of the map shown in Figure 4. The background image shows the line-of-sight magnetic field measured by HMI, with red as positive and blue as negative polarities. The range of the magnetic field is from -80 to 80 Gauss.

From the full-disk wave-speed perturbation and flow maps obtained every eight hours, we select an area $13.2^{\circ}$ wide in longitude, i.e. $-6.6^{\circ}$ to $6.6^{\circ}$ from the central meridian, to construct the synoptic wave-speed perturbation and flow maps. Since the Carrington rotation rate corresponds to a shift of approximately $4.4^{\circ}$ every eight hours, each location in the constructed synoptic maps is averaged roughly three times (i.e. one whole day). The resultant synoptic map for each depth consists of $3000 \times 1000$ pixels. Since such a map is difficult to display, we show in Figure 6 a binned-down synoptic large-scale flow map obtained for the depth of 1-3 Mm for Carrington Rotation 2097 during the period from 20 May to 16 June, 2010. The vectors in the figure are obtained by averaging the flow fields in areas of $15^{\circ} \times 15^{\circ}$ with a $3^{\circ}$ sampling rate. The maps of this type are similar to the subsurface flow maps obtained from the ring-diagram analysis (Haber et al., 2002). From Figure 6, it can be found that the large-scale flows often converge around magnetic regions.

Normally, the full-disk flow maps and the synoptic flow maps are provided as residual flow maps after subtracting the flows averaged over the entire Carrington rotation. The subtracted average maps contain the differential rotation, meridional flows, and possibly some systematic effects. Figure 7 shows examples of the subsurface differential rotation speed and meridional flow speed obtained by averaging the calculations for Carrington Rotation 2097. These results are also provided online together with the synoptic flow maps. 


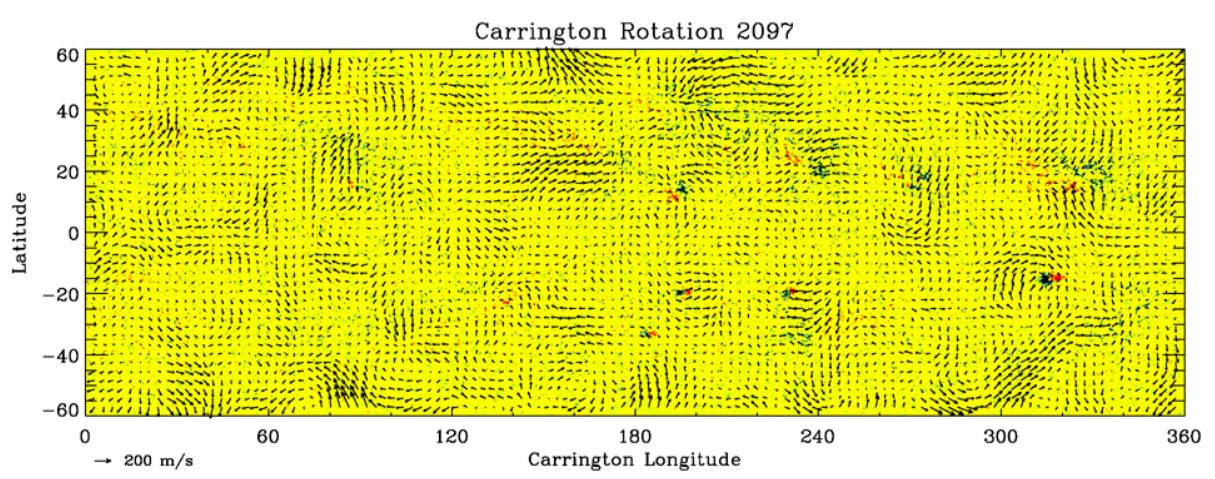

Figure 6 Synoptic map of large-scale horizontal flows at the depth of 1-3 Mm for CR 2097. The background image is the corresponding line-of-sight magnetic field, with red as positive and blue as negative polarities, in the range of -50 to 50 Gauss.

Figure 7 Averaged rotation (upper) and meridional flow speeds (lower) at selected depths for Carrington Rotation 2097. The rotation speed is relative to the constant Carrington rotation rate.
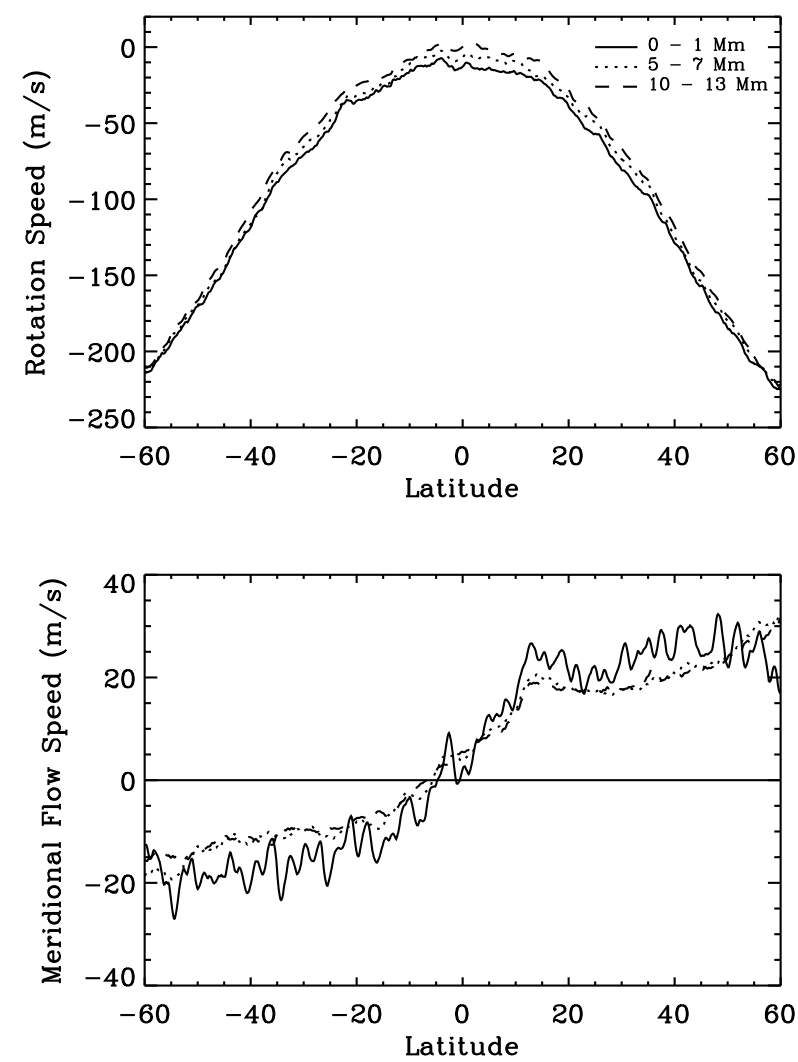

\subsection{Target Areas}

As already mentioned in Section 2, the pipeline can also be run for specific target areas and specific time intervals. The pipeline users are required to provide the Carrington coordinates of the center of the target area, and the mid-time of the time interval. 


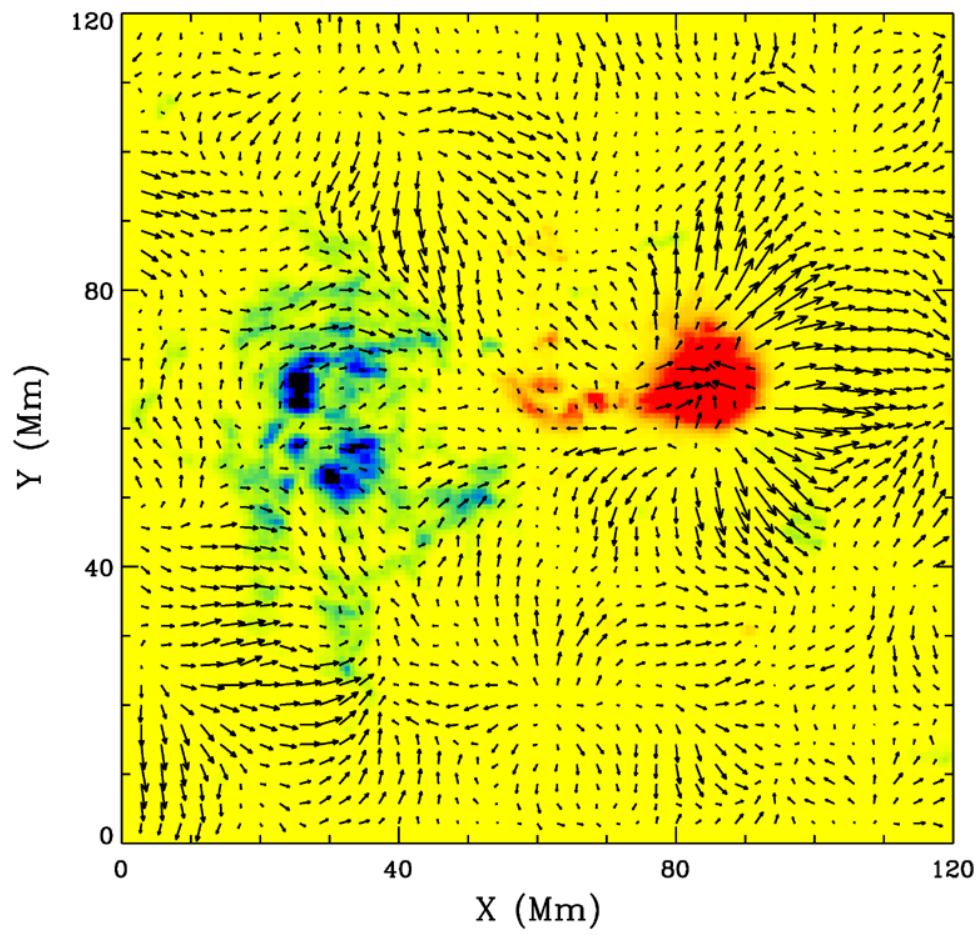

Figure 8 Subsurface flow fields of AR 11072 at the depth of 1-3 Mm, obtained from 16:00-23:59 UT 23 May 2010. The background image is line-of-sight magnetic field, with red positive and blue negative. The image is displayed with a scale from -1000 to 1000 Gs. The arrows are displayed after a $2 \times 2$ rebinning, and the longest arrow represents a speed of $300 \mathrm{~m} \mathrm{~s}^{-1}$.

Figure 8 shows an example of a small part (approximately 1/9) of a target area, with an active region, AR 11072, included in it. The subsurface flow field, at the depth of $1-3 \mathrm{Mm}$, is displayed after a $2 \times 2$ rebinning. The displayed time period, 16:00-23:59 UT on 23 May 2010 , is approximately 2.5 days after the start of emergence of this active region that was still growing. Our results clearly show subsurface outflows around the leading sunspot, and some converging flows inside it. Comparing with the previous results of Kosovichev (2009) and Zhao, Kosovichev, and Sekii (2010), one may conclude that the subsurface flow fields of active regions evolve with the evolution of active regions. There may be prominent outflows around sunspots during their early growing phase and also their decaying phase, but there may also be strong converging flows during the stable period. Our continuous monitoring of the solar full-disk subsurface flows may give us an opportunity to statistically study the changes of the sunspot's subsurface flows with the sunspot evolution.

\section{Summary}

We have developed a time-distance helioseismology data-analysis pipeline for SDO/HMI Doppler observations. This pipeline performs acoustic travel-time measurements based on two different methods, and conducts inversions based on two different sensitivity kernels calculated in the ray-path and Born approximations. The pipeline gives nearly real-time 
routine products of full-disk wave-speed perturbations and flow-field maps in the range of depth $0-20 \mathrm{Mm}$ every eight hours, and provides the corresponding synoptic wave-speed perturbation and flow-field maps for each Carrington rotation. The averaged rotation speed and meridional flow speed are also provided separately for each rotation. In addition to these routine production, the pipeline can also be used for analysis of specific target areas for specific time intervals. This data-analysis pipeline will provide important information about the subsurface structure and dynamics on both local and global scales, and its continuous coverage through years to come will be useful for understanding the connections between solar subsurface properties and magnetic activity in the chromosphere and corona. With the improvement of our understanding of acoustic-wave and magnetic-field interactions, and also the measurement and inversion techniques, the pipeline codes will be regularly revised.

\section{References}

Beck, J.G., Gizon, L., Duvall, T.L. Jr., 2002, Astrophys. J. Lett. 575, L47.

Birch, A.C., Gizon, L.: 2007, Astron. Nachr. 328, 228.

Birch, A.C., Kosovichev, A.G.: 2000, Solar Phys. 192, 193.

Birch, A.C., Kosovichev, A.G., Duvall, T.L. Jr., 2004, Astrophys. J. 608, 580.

Chou, D.-Y., Dai, D.-C.: 2001, Astrophys. J. Lett. 559, L175.

Couvidat, S., Birch, A.C., Kosovichev, A.G.: 2006, Astrophys. J. 640, 516.

Couvidat, S., Birch, A.C., Kosovichev, A.G., Zhao, J.: 2004, Astrophys. J. 607, 554.

Couvidat, S., Gizon, L., Birch, A.C., Larsen, R.M., Kosovichev, A.G.: 2005, Astrophys. J. Suppl. 158, 217.

Couvidat, S., Zhao, J., Birch, A.C., Kosovichev, A.G., Duvall, T.L. Jr., Parchevsky, K.V., Scherrer, P.H.: 2010, Solar Phys. doi:10.1007/s11207-010-9652-y.

Duvall, T.L. Jr., Gizon, L.: 2000, Solar Phys. 192, 177.

Duvall, T.L. Jr., Jefferies, S.M., Harvey, J.W., Pomerantz, M.A.: 1993, Nature 362, 430.

Duvall, T.L. Jr., D’Silva, S., Jefferies, S.M., Harvey, J.W., Schou, J.: 1996, Nature 379, 235.

Duvall, T.L. Jr., Kosovichev, A.G., Scherrer, P.H., Bogart, R.S., Bush, R.I., DeForest, C., Hoeksema, J.T., Schou, J., Saba, J.L.R., Tarbell, T.D., Title, A.M., Wolfson, C.J., Milford, P.N.: 1997, Solar Phys. 170, 63.

Georgobiani, D., Zhao, J., Kosovichev, A.G., Bensen, D., Stein, R.F., Nordlund, Å.: 2007, Astrophys. J. 657, 1157.

Giles, P.M., Duvall, T.L. Jr., Scherrer, P.H., Bogart, R.S.: 1997, Nature 390, 52.

Gizon, L., Birch, A.C.: 2002, Astrophys. J. 571, 966.

Gizon, L., Duvall, T.L. Jr., Larsen, R.M.: 2000, J. Astrophys. Astron. 21, 339.

Gizon, L., Schunker, H., Baldner, C.S., Basu, S., Birch, A.C., Bogart, R.S., Braun, D.C., Cameron, R., Duvall, T.L. Jr., Hanasoge, S.M., Jackiewicz, J., Roth, M., Stahn, T., Thompson, M.J., Zharkov, S.: 2009, Space Sci. Rev. 144, 249.

Haber, D.A., Hindman, B.W., Toomre, J., Bogart, R.S., Larsen, R.M., Hill, F.: 2002, Astrophys. J. 570, 885.

Hanasoge, S.M., Couvidat, S., Rajaguru, S.P., Birch, A.C.: 2008, Mon. Not. Roy. Astron. Soc. 391, 1931.

Ilonidis, S., Zhao, J., Hartlep, T.: 2009, Solar Phys. 258, 181.

Jacobsen, B.H., Møller, I., Jensen, J.M., Effersø, F.: 1999, Phys. Chem. Earth, Part A, Solid Earth Geod. $24,15$.

Jackiewicz, J., Gizon, L., Birch, A.C.: 2008, Solar Phys. 251, 381.

Jensen, J.M., Duvall, T.L. Jr., Jacobsen, B.H.: 2003, In: Sawaya-Lacoste, H. (ed.) Proc. SOHO 12/ GONG+ 2002, Local and Global Helioseismology: the Present and Future SP-517, ESA, Noordwijk, 315.

Jensen, J.M., Duvall, T.L. Jr., Jacobsen, B.H., Christensen-Dalsgaard, J.: 2001, Astrophys. J. Lett. 553, L193.

Jensen, J.M., Jacobsen, B.H., Christensen-Dalsgaard, J.: 2000, Solar Phys. 192, 231.

Kitiashvili, I.N., Kosovichev, A.G., Mansour, N.N., Wray, A.A.: 2011, Solar Phys. 268, 283.

Komm, R., Corbard, T., Durney, B.R., Hernandez Gonzalez, I., Hill, F., Howe, R., Toner, C: 2004, Astrophys. J. 605, 554 .

Kosovichev, A.G.: 1996, Astrophys. J. Lett. 461, L55.

Kosovichev, A.G.: 2009, Space Sci. Rev. 144, 175.

Kosovichev, A.G.: 2010, Solar Phys. submitted. arXiv:1010.4927.

Kosovichev, A.G., Duvall, T.L. Jr.: 1997, In: Pijers, F.P., Christensen-Dalsgaard, J., Rosenthal, C.S. (eds.) Proceedings of SCORe'96: Solar Convection and Oscillations and Their Relationship, Astrophysics and Astronomy Library 225, Kluwer, Dordrecht, 241. 
Kosovichev, A.G., Duvall, T.L. Jr., Scherrer, P.H.: 2000, Solar Phys. 192, 159.

Lindsey, C., Braun, D.C.: 2000, Solar Phys. 192, 261.

Lindsey, C., Braun, D.C.: 2005a, Astrophys. J. 620, 1107.

Lindsey, C., Braun, D.C.: 2005b, Astrophys. J. 620, 1118.

Nigam, R., Kosovichev, A.G.: 2010, Astrophys. J. 708, 1475.

Parchevsky, K.V., Zhao, J., Kosovichev, A.G.: 2008, Astrophys. J. 678, 1498.

Rajaguru, S.P., Birch, A.C., Duvall, T.L. Jr., Thompson, M.J., Zhao, J.: 2006, Astrophys. J. 646, 543.

Rempel, M., Schüssler, M., Knölker, M.: 2009, Astrophys. J. 691, 640.

Sekii, T., Kosovichev, A.G., Zhao, J., Tsuneta, S., Shibahashi, H., Berger, T.E., Ichimoto, K., Katsukawa, Y., Lites, B., Nagata, S., Shimizu, T., Shine, R.A., Suematsu, Y., Tarbell, T.D., Title, A.M.: 2007, Publ. Astron. Soc. Japan 59, S637.

Scherrer, P.H., Bogart, R.S., Bush, R.I., Hoeksema, J.T., Kosovichev, A.G., Schou, J., Rosenberg, W., Springer, L., Tarbell, T.D., Title, A., Wolfson, C.J., Zayer, I., MDI Engineering Team: 1995, Solar Phys. 162, 129.

Schou, J., Scherrer, P.H., Watchter, R., Couvidat, S., Bush, R.I.: et al.: 2011, Solar Phys. in preparation.

Schunker, H., Braun, D.C., Cally, P.S., Lindsey, C.: 2005, Astrophys. J. Lett. 621, L149.

Snodgrass, H.B., Ulrich, R.K.: 1990, Astrophys. J. 351, 309.

Stein, R.F., Lagerfjärd, A., Nordlund, Å., Georgobiani, D.: 2011, Solar Phys. 268, 271.

Zhao, J.: 2007, Astrophys. J. Lett. 664, L139.

Zhao, L., Jordan, T.H.: 1998, Geophys. J. Int. 133, 683.

Zhao, J., Kosovichev, A.G.: 2004, Astrophys. J. 603, 776.

Zhao, J., Kosovichev, A.G.: 2006, Astrophys. J. 643, 1317.

Zhao, J., Kosovichev, A.G., Duvall, T.L. Jr.: 2001, Astrophys. J. 557, 384.

Zhao, J., Kosovichev, A.G., Sekii, T.: 2010, Astrophys. J. 708, 304.

Zhao, J., Georgobiani, D., Kosovichev, A.G., Benson, D., Stein, R.F., Nordlund, Å.: 2007, Astrophys. J. 659, 848 .

Zhao, J., Hartlep, T., Kosovichev, A.G., Mansour, N.N.: 2009, Astrophys. J. 702, 1150.

Zharkov, S., Thompson, M.J.: 2008, Solar Phys. 251, 369. 